\title{
Fonctions du conte tournierien dans La Goutte d'or
}

\author{
Beatrice Ness \\ (Marquette University)
}

Le "romancier" Michel Tournier a toujours écrit des contes : c'est à partir de Vendredi ou les limbes du Pacifique (1967) qu'il modela, en conteur palimpsestueux, Vendredi ou la vie sauvage (1971); il transforma aussi Le Roi des Aulnes (1970) pour donner naissance à son modèle archétypal : Gilles et Jeanne (1983)'; enfin, c'est par un recueil de brefs récits : Le Coq de bruyère (1978) que Tournier s'affirma définitivement comme "conteur"--"conteur", précise-t-il lors de plusieurs interviews, et non pas "nouvelliste"--appellation qu'il réprouve! En effet, dans son processus de genèse romanesque, Tournier refuse systématiquement la "bassesse" réaliste de la nouvelle, et focalise toute son attention sur le conte qui réactive les mythes. Romancier aux origines philosophiques, il s'explique en ces termes, dans Le Vent Paraclet, sur l'utilisation du conte dans ses écrits : "le passage de la métaphysique au roman devait m'être fourni par un mythe, c'est-à-dire une histoire fondamentale" (188). Peut-être ceci explique-t-il en partie cette profusion de petits contes intercalés dans la trame romanesque ou réécrits à partir de ces romans mêmes. Continuant à disserter, dans Le Vent Paraclet, sur la dimension mythologique de ses oeuvres, Tournier ajoute que le mythe est au premier degré "une histoire pour enfants" (1988) : il insiste ainsi sur le caractère de perfection du conte puisqu'à son sens un texte ne peut être adressé aux enfants que s'il est parfait. Enfin, l'auteur conclut en se tournant vers le lecteur adulte et précise que le mythe est "une histoire que tout le monde connaît déjà" (189) : 1'acte de lecture étant, pour les lecteurs que nous sommes, adjacent à notre expérience, il s'assimile à une reconnaissance. ${ }^{2}$ A partir de ces références archétypales de perfection et de reconnaissance, Tournier établit alors, dans Le Vol du vampire (35-37), trois catégories de récits auxquels il attribue les valeurs hiérarchiques suivantes : Au premier échelon se situe "la nouvelle" s'affirmant par son réalisme et que Tournier qualifie d' "opaque"; au second degré, "la fable" livre une moralité explicite et correspond au "transparent"; et enfin, au niveau ultime, Tournier place "le conte" qui par son "instruction cachée s'apparente au "translucide". 
C'est peut-être cette certitude, chez Tournier, de la surprématie du conte, qui le pousse à utiliser, une fois de plus, cette forme dans son roman de $1986: L a$ Goutte d'or. Rien de plus curieux, en effet, que l'insertion de ces deux petits contes : "Barberousse" et "La Reine blonde"--qui semblent à première vue plaqués--dans ce long roman picaresque. L'auteur nous y relate les péripéties de son "picaro", Idriss, petit oasien berbère, qui décide de s'arracher à son Sahara natal pour aller vers Paris, à la recherche de sa photo qu'une femme blonde, surgissant d'une Land Rover, avait prise. Bien entendu, Idriss n'a jamais reçu la photo dont cette dernière lui avait promis l'envoi. Encouragé par la croyance en l'esprit belbali qui veut que la photo soit maléfique si on ne la possède pas, Idriss décide de partir vers le nord. Au moment même de cette prise de décision, un premier conte enchâssé : "Barberousse ou le portrait du roi" est inséré par le prétexte des noces berbères. Des questions cruciales se posent alors au lecteur en ce qui concerne le fonctionnement du récit : pourquoi Tournier arrête-t-il son roman picaresque pour y insérer ce premier conte? Et pour quelles raisons l'auteur persiste-t-il et réitère-t-il sa tentative, en nous soumettant, en fin de roman, un autre conte, enchâssé dans le roman : "La Reine blonde"? Quels sont les effets recherchés et qu'apporte l'intrusion de ces deux contes à la dynamique romanesque? Autant de questions auxquelles cette courte étude va tenter de répondre en mettant en valeur, à partir du corpus de La Goutte d'or, les trois fonctions primordiales du conte tournierien.

Une première fonction diégétique nous est livrée à partir du premier conte intercalé dans le roman. Cette véridique histoire de l'aventurier musulman du quatorzième siècle, Kheir ed Dîn, surnommé "Barberousse", n'est pas sans rappeler, du reste, "Barbedor"--ce roi arabe dont l'histoire nous est narrée en un bref conte inséré dans l'imposant roman des Rois Mages: Gaspard, Melchior et Balthazar. Mais, à l'encontre de Barbedor qui, fier de la blonde couleur de sa barbe, n'a cesse de la protéger par une "housse à barbe", Barberousse, pour sa part, a en horreur sa pilosité-le roux étant méprisé en Islam. C'est le portrait, "cette symphonie en roux majeur" (52) que fait de lui Kerstine, artiste scandinave, qui le réconcilie avec lui-même et avec le monde.

Ce conte qu'écoute le narrataire Idriss suspend le temps diégétique à des fins narratives : ce conte permet au héros Idriss de prendre conscience de l'urgence de son départ; et, de même que Barberousse trouve la délivrance et la guérison en voyant son portrait, Idriss se doit de partir pour trouver sa photo. Ainsi se dégage la première fonction--fonction éclairante--de ce petit conte mis en parallèle à la situation complexe du roman : l'aventure mythique de "Barberousse" évoquée par Abdullah Fehr, conteur noir venu des confins du Soudan et du Tibesti, libère le héros et l'engage à partir : "Partir, pense Idriss, 
prendre la route du nord [...] c'était ce que lui conseillait [...] la légende de Kheir ed Dîn" (65). Ce premier conte supporte donc la charpente narrative du roman puisqu'il permet au héros de prendre conscience de ce qu'il doit faire : ainsi, la légende de "Barberousse" bloque le roman, le diffère, afin de mieux l'expliquer, de mieux l'éclairer.

Mais à "Barberousse"--véritable prolepse narrative--répond dans le récit une seconde prolepse, un second conte : "La Reine blonde", et qui introduit la seconde fonction du conte tournierien : la fonction dynamique.

"Tout est signe" (15) avoue Abel Tiffauges dans Le Roi des Aulnes. Et en effet, si ces contes sont des signes pour le héros, ils lui permettent, à distance, d'observer et de refléter la situation complexe dans laquelle il se trouve impliqué. Ainsi le confesse lui-même l'auteur : "Chez moi [...] c'est le mécanisme mythologique et symbolique qui est si contraignant qu'il détermine entièrement l'action des personnages". ${ }^{3}$ Embrayeur de l'action, déclencheur du départ, le premier conte "Barberousse" donne au héros sa liberté. Délivrance certes, mais en vue d'une guérison: c'est le second conte--seconde prolepse narrative--qui dynamise le récrit en proposant à Idriss une solution à son problème--de là, cette seconde légende mise en contrepoint du premier récit spéculaire--et qui revitalise la structure romanesque du texte. Ces deux contes deviennent alors les deux ponts dans l'articulation narrative en ce que leurs occurrences constituent un véritable paradigme. En effet, "Barberousse" et "La Reine blonde" relèvent des mêmes motifs : ils traitent essentiellement de couleurs néfastes (le roux, le blond) et du rôle de ces couleurs dans l'image. Ainsi, dans "Barberousse", le roi est roux et cache sa honte sous un turban et une "housse à barbe". De même, "La Reine blonde" qui, après l'assassinat du roi par jalousie, comprend le danger que représente sa chevelure, décide de vivre voilée jusqu'au jour de sa mort. La mise en parallèle des deux contes dans leur enchaînement syntagmatique fait apparaître des paradigmes qui dégagent les correspondances structurelles, non seulement entre les deux contes, mais aussi dans la trame même du roman La Goutte d'or.

Le schéma structurel pourrait se réduire aux quatre séquences suivantes : 
CONTE 1

"Barberousse"
ROMAN

La Goutte d'or
CONTE 2

"La Reine blonde"

\begin{tabular}{|c|c|c|c|}
\hline $1^{\circ}$ & le $\frac{\text { ROUX }}{1}$ (est honteux) & $\rightarrow l^{\prime} \underline{O R}$ (est maléfique) & $\rightarrow$ le $\frac{\text { BLOND }}{l}$ (est honteux) \\
\hline $2^{2^{\circ}}$ & $\begin{array}{l}\frac{\downarrow}{\text { PORTRAIT du roi }} \\
\text { (fait sans qu'il } \\
\text { le sache) }\end{array}$ & $\frac{\text { PHOTO d'Idriss }}{\text { (prise sur le vif) }}$ & $\begin{array}{l}\text { PORTRAIT de la reine } \\
\text { (fait sans qu'elle } \\
\text { le sache) }\end{array}$ \\
\hline $\begin{array}{c}3^{\circ} \\
\text { L'ACTION }\end{array}$ & $\begin{array}{l}\text { BENEF IQUE } \\
\text { EBLOUISSEMENT }\end{array}$ & $\begin{array}{l}\text { MALEFIQUE } \\
\text { - recherche ACTIVE }\end{array}$ & $\begin{array}{l}\text { MALEF IQUE } \\
\text { EFFACEMENT }\end{array}$ \\
\hline $\begin{array}{c}4^{\circ} \\
\text { LE SIGNE }\end{array}$ & & $\begin{array}{l}\text { CALLIGRAPHIE } \\
\text { ABSOLUE }\end{array}$ & $\mho_{\text {CALLIGRAPHIE }}$ \\
\hline
\end{tabular}

Le parallélisme des deux contes équilibre alors le roman et lui confère son unité structurelle : le symbole même de la photo se trouve être le répondant romanesque aux portraits des contes. En effet, aux deux portraits, bénéfique ("Barberousse") et néfaste ("La Reine blonde"), correspond le dépliement photographique de la première image d'Idriss qui multiplie le maléfice : photos tronquées des touristes de Mustapha sur décor de carton-pâte; photo judiciaire d'Idriss, modifiée par son nez sanglant et son oeil au beurre noir; photos des mannequins de Milan, "images d'images" (211), sur fond vrai--figures irréelles inversant les photos de Mustapha; et enfin la photo d'identité d'Idriss, photo faussée puisqu'Idriss reçoit, dans la cabine automatique, l'image d'un barbu qui n'est pas lui, et qui reprend le portrait initial de "Barberousse" :

Idriss attendit encore. Deux nouvelles images tombèrent : celles d'un homme barbu. Il se regarda longuement dans le miroir fêlé de la cabine. Après tout pourquoi n'aurait-il pas eu une barbe avant de quitter Tabelbala? Les barbus ont aussi droit à un passeport (108-109).

Ces déplacements photographiques répondent au paradigme des deux contes et par leur symbolisme ouvrent la voie à la troisième fonction des contes : une fonction salvatrice.

Fonction salvatrice, en effet, puisque "La Reine blonde" transmet au héros un signe, code symbolique qui permettra à Idriss de sortir de "la souille" dans laquelle il est tombé. Ainsi, à la fin du conte "La Reine blonde", le portrait 
maléfique de la reine tombe aux mains du pêcheur Antar. Afin de mettre fin à l'envoûtement auquel le portrait soumet Antar, Riad, son fils, s'initie à l'art plein de sagesse de la calligraphie. La superposition des arabesques calligraphiques au portrait de la reine fera apparaître la vérité intelligible qu'exprime l'ensemble de signes constituant ce visage. Ainsi, Riad fait découvrir à son père, le pêcheur, "la complainte de la reine blonde victime de sa propre beauté" (251) :

...mon père, je suis venu avec mes calames, s'écrie Riad, mon encre et mes parchemins pour déchiffrer la signification de la reine blonde. Et voici ce que j'ai trouvé. Il éleva vers le ciel les huit feuillets superposés qu'il avait couverts de signes calligraphiés. Aussitôt apparut le visage de la reine, un visage composé d'arabesques, un visage translucide, apaisé, spiritualisé (251).

Par cette recherche mystique sera sauvé le pêcheur et mis en abîme le roman tout entier. Car si la calligraphie sauve le pêcheur, la morale du conte se résume comme suit : écrivez et vous serez sauvé; le roman entier La Goutte d'or est alors mis en abîme comme expérience ultime de sauvetage. En effet, de même que la calligraphie offre au pêcheur Antar, par l'intermédiaire de son fils Riad, une initiation spirituelle salvatrice, c'est aussi par le signe calligraphique qu'Idriss se détachera des images avilissantes et corruptrices. Présenté au maître calligraphe Abd Al Gahafari, Idriss comprend que "la calligraphie est libération" (234) et que la guérison lui sera livrée par la goutte, d'or, antidote de l'asservissement par l'image. Le conte "La Reine blonde" magnifiant le principe calligraphique entrepris par Idriss, s'affirme alors comme exemplaire. Le conte invite, comme nous l'explique Michel Butor dans "La Balance des fées", à réagir sur [la réalité], nous invite à la transformer, à remettre à l'endroit ce qui, en elle, est mal placé" (65). De là s'éclaire la fin du roman où Tournier, reprenant l'épisode initial du premier chapitre--la mort jubilatoire d'Ibrahim--permet à Idriss d'atteindre l'exaltation mystique de ce que l'auteur appelle : "l'humour blanc". En effet, l'écrivain accomplit, dans cet excipit romanesque, l'ambition du Vent Paraclet : atteindre à "un cosmique comique". Idriss, muni de son marteau-piqueur, fend la vitrine de Cristobal \& Co où se trouve exposée la goutte d'or, "symbole de libération" (256) et d'absolu. Alors Tournier touche au "naturalisme mystique" qu'il prétend rechercher dans tous ses romans : en effet, si le sujet de La Goutte d'or est certes bien ancré dans le réel par le problème des immigrés maghrébins, la technique romanesque employée renvoie néanmoins à une métaphysique exprimée par des signes que nous expliquent les deux contes insérés. Les 
mythes que nous proposent ces deux contes provoquent donc l'ouverture polysémique du roman : car comment s'achève réellement l'histoire d'Idriss? L'ambiguïté narrative de la fin du roman est renforcée par la multiplicité sémique des signes employés par l'auteur, dont le propos n'est donc pas de nous livrer une vision réaliste du monde mais bien d'interpréter des mythes et des symboles à des fins salvatrices : 1'humour blanc auquel est en proie Idriss, à la dernière image, délie le sortilège auquel le héros se trouvait lié, et lui permet ainsi de dépasser sa misérable condition humaine :

Dansant sur place avec son marteau-piqueur, [Idriss] ne vit pas la vitrine de CRISTOBAL \& Co se fendre de haut en bas. Il n'entendit pas le hululement de la sirène d'alerte déclenchée par les palpeurs sismiques. Ding, ding, ding. Idriss danse toujours avec en tête une fantasmagorie de libellules, de criquets et de bijoux agités d'une trépidation forcenée [...] Sourd et aveugle, Idriss continue à danser devant la goutte d'or avec sa cavalière pneumatique (257).

Ainsi, en utilisant ces deux contes aux fins closes et claires pour expliciter un roman : La Goutte d'or, à la fin ouverte, l'auteur ne cherche qu'à stimuler la participation du lecteur afin que ce dernier retrouve les vérités cachées derrière les lignes du roman. Les trois fonctions du conte tournierien : éclairante, dynamique et salvatrice, correspondent donc bien à ce que l'auteur recherche : "Un bon conte, confesse-t-il, est supérieur à tous les romans, car il a les qualités par excellence de la littérature [...] la brièveté, la limpidité, le fondamental". ${ }^{4}$ Reste au lecteur de savoir décrypter, par l'intermédiaire de ces deux contes, le message romanesque.

\section{NOTES}

C'est en tout cas ce que soutient--et prouve!--Susan Petit dans son article : "Gilles et Jeanne : Tournier's Le Roi des Aulnes Revisited".

2

Cette notion de lecture innocente/lecture savante est développée par Joseph H. McMahon dans son article : "Michel Tournier's Texts for Children".

C'est ce qu'il confesse à Serge Koster dans son récent livre sur Michel Tournier (p. 32). 
C'est ce qu'il affirme dans une célèbre interview du Monde du 2 décembre 1979 , où il dit avoir toujours voulu être écrivain voyant (p. 8).

\section{Oeuvres citées}

Butor, Michel. "La Balance des fées." Répertoire. Paris : Minuit, 1960, pp. 60-74.

Koster, Serge. Michel Tournier. Paris : Ed. Veyrier, 1986.

McMahon, Joseph H. "Michel Tournier's Texts for Children." Children's Literature, Vol. 13, 1985, pp. 154-169.

Petit, Susan. "Gilles et Jeanne : Tournier's Le Roi des Aulnes Revisited." Romanic Review, May 1985, pp. 306-315.

Tournier, Michel. Vendredi ou les limbes du Pacifique. Paris: Gallimard, 1967.

-- Le Roi des Aulnes. Paris : Gallimard, 1970.

-- Vendredi ou la vie sauvage. Paris : Gallimard, 1971.

-- Le Vent Paraclet. Paris : Gallimard, 1977.

-- Le Coq de bruyère. Paris : Gallimard, 1978.

-- Gaspard, Melchior et Balthazar. Paris : Gallimard, 1980.

-- Le Vol du vampire. Paris : Mercure de France, 1981.

-- Gilles et Jeanne. Paris : Gallimard, 1983.

-- La Goutte d'or. Paris : Gallimard, 1986.

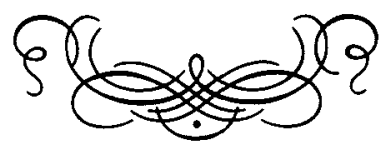

